\title{
The Physical Science Behind Climate Change
}

\author{
By William Collins, Robert Colman, James Haywood, Martin R. Manning and Philip Mote
}

The authors were participants in Working Group I of the 2007 IPCC assessment. William Collins is a professor in residence in the department of earth and planetary science at the University of California, Berkeley, and a senior scientist at Lawrence Berkeley National Laboratory and the National Center for Atmospheric Research in Boulder, Colo. Robert Colman is a senior research scientist in the Climate Dynamics Group at the Australian Bureau of Meteorology Research Center in Melbourne. James Haywood is the manager of aerosol research in the Observational Based Research Group and the Chemistry, Climate and Ecosystem Group at the Met Office in Exeter, England. Martin R. Manning is director of the IPCC WG I Support Unit at the NOAA Earth System Research Laboratory in Boulder, Colo. Philip Mote is the Washington State climatologist, a research scientist in the Climate Impacts Group at the University of Washington, and an affiliate professor in the department of atmospheric sciences.

Why are climatologists so highly confident that human activities are dangerously warming the earth? Here some of the participants in the most recent and comprehensive international review of the scientific evidence summarize the arguments and discuss what uncertainties remain.

For a scientist studying climate change, "eureka" moments are unusually rare. Instead progress is generally made by a painstaking piecing together of evidence from every new temperature measurement, satellite sounding or climate-model experiment. Data get checked and rechecked, ideas tested over and over again. Do the observations fit the predicted changes? Could there be some alternative explanation? Good climate scientists, like all good scientists, want to ensure that the highest standards of proof apply to everything they discover.

And the evidence of change has mounted as climate records have grown longer, as our understanding of the climate system has improved and as climate models have become ever more reliable. Over the past 20 years, evidence that humans are affecting the climate has accumulated inexorably, and with it has come ever greater certainty across the scientific community in the reality of recent climate change and the potential for much greater change in the future. This increased certainty is starkly reflected in the latest report of the Intergovernmental Panel on Climate Change (IPCC), the fourth in a series of assessments of the state of knowledge on the topic, written and reviewed by hundreds of scientists worldwide.

The panel released a condensed version of the first part of the report, on the physical science basis of climate change, in February. Called the "Summary for Policymakers," it delivered to policymakers and ordinary people alike an unambiguous message: scientists are more confident than ever that humans have interfered with the climate and that further human-induced climate change is on the way. Although the report finds that some of these further changes are now inevitable, its analysis also confirms that the future, particularly in the longer term, remains largely in our hands-the magnitude of expected change depends on what humans choose to do about greenhouse gas emissions. 
The physical science assessment focuses on four topics: drivers of climate change, changes observed in the climate system, understanding cause-and-effect relationships, and projection of future changes. Important advances in research into all these areas have occurred since the IPCC assessment in 2001. In the pages that follow, we lay out the key findings that document the extent of change and that point to the unavoidable conclusion that human activity is driving it.

\section{Drivers of Climate Change}

Atmospheric concentrations of many gases-primarily carbon dioxide, methane, nitrous oxide and halocarbons (gases once used widely as refrigerants and spray propellants) - have increased because of human activities. Such gases trap thermal energy (heat) within the atmosphere by means of the wellknown greenhouse effect, leading to global warming. The atmospheric concentrations of carbon dioxide, methane and nitrous oxide remained roughly stable for nearly 10,000 years, before the abrupt and rapidly accelerating increases of the past 200 years. Growth rates for concentrations of carbon dioxide have been faster in the past 10 years than over any 10-year period since continuous atmospheric monitoring began in the 1950s, with concentrations now roughly 35 percent above preindustrial levels (which can be determined from air bubbles trapped in ice cores). Methane levels are roughly two and a half times preindustrial levels, and nitrous oxide levels are around 20 percent higher.

How can we be sure that humans are responsible for these increases? Some greenhouse gases (most of the halocarbons, for example) have no natural source. For other gases, two important observations demonstrate human influence. First, the geographic differences in concentrations reveal that sources occur predominantly over land in the more heavily populated Northern Hemisphere. Second, analysis of isotopes, which can distinguish among sources of emissions, demonstrates that the majority of the increase in carbon dioxide comes from combustion of fossil fuels (coal, oil and natural gas). Methane and nitrous oxide increases derive from agricultural practices and the burning of fossil fuels.

Climate scientists use a concept called radiative forcing to quantify the effect of these increased concentrations on climate. Radiative forcing is the change that is caused in the global energy balance of the earth relative to preindustrial times. (Forcing is usually expressed as watts per square meter.) A positive forcing induces warming; a negative forcing induces cooling. We can determine the radiative forcing associated with the long-lived greenhouse gases fairly precisely, because we know their atmospheric concentrations, their spatial distribution and the physics of their interaction with radiation.

Climate change is not driven just by increased greenhouse gas concentrations; other mechanisms-both natural and human-induced-also play a part. Natural drivers include changes in solar activity and large volcanic eruptions. The report identifies several additional significant human-induced forcing mechanisms - microscopic particles called aerosols, stratospheric and tropospheric ozone, surface albedo (reflectivity) and aircraft contrails—although the influences of these mechanisms are much less certain than those of greenhouse gases.

Investigators are least certain of the climatic influence of something called the aerosol cloud albedo effect, in which aerosols from human origins interact with clouds in complex ways and make the clouds brighter, reflecting sunlight back to space. Another source of uncertainty comes from the direct effect of aerosols from human origins: How much do they reflect and absorb sunlight directly as particles? Overall these aerosol effects promote cooling that could offset the warming effect of long-lived greenhouse gases to some extent. But by how much? Could it overwhelm the warming? Among the advances achieved 
since the 2001 IPCC report is that scientists have quantified the uncertainties associated with each individual forcing mechanism through a combination of many modeling and observational studies. Consequently, we can now confidently estimate the total human-induced component. Our best estimate is some 10 times larger than the best estimate of the natural radiative forcing caused by changes in solar activity.

This increased certainty of a net positive radiative forcing fits well with the observational evidence of warming discussed next. These forcings can be visualized as a tug-of-war, with positive forcings pulling the earth to a warmer climate and negative ones pulling it to a cooler state. The result is a no contest; we know the strength of the competitors better than ever before. The earth is being pulled to a warmer climate and will be pulled increasingly in this direction as the "anchorman" of greenhouse warming continues to grow stronger and stronger.

\section{Observed Climate Changes}

The many new or improved observational data sets that became available in time for the 2007 IPCC report allowed a more comprehensive assessment of changes than was possible in earlier reports. Observational records indicate that 11 of the past 12 years are the warmest since reliable records began around 1850 . The odds of such warm years happening in sequence purely by chance are exceedingly small. Changes in three important quantities-global temperature, sea level and snow cover in the Northern Hemisphere-all show evidence of warming, although the details vary. The previous IPCC assessment reported a warming trend of $0.6 \pm 0.2$ degree Celsius over the period 1901 to 2000. Because of the strong recent warming, the updated trend over 1906 to 2005 is now $0.74 \pm 0.18$ degree $\mathrm{C}$. Note that the 1956 to 2005 trend alone is $0.65 \pm 0.15$ degree $\mathrm{C}$, emphasizing that the majority of 20th-century warming occurred in the past 50 years. The climate, of course, continues to vary around the increased averages, and extremes have changed consistently with these averages-frost days and cold days and nights have become less common, while heat waves and warm days and nights have become more common.

The properties of the climate system include not just familiar concepts of averages of temperature, precipitation, and so on but also the state of the ocean and the cryosphere (sea ice, the great ice sheets in Greenland and Antarctica, glaciers, snow, frozen ground, and ice on lakes and rivers). Complex interactions among different parts of the climate system are a fundamental part of climate change-for example, reduction in sea ice increases the absorption of heat by the ocean and the heat flow between the ocean and the atmosphere, which can also affect cloudiness and precipitation.

A large number of additional observations are broadly consistent with the observed warming and reflect a flow of heat from the atmosphere into other components of the climate system. Spring snow cover, which decreases in concert with rising spring temperatures in northern midlatitudes, dropped abruptly around 1988 and has remained low since. This drop is of concern because snow cover is important to soil moisture and water resources in many regions.

In the ocean, we clearly see warming trends, which decrease with depth, as expected. These changes indicate that the ocean has absorbed more than 80 percent of the heat added to the climate system: this heating is a major contributor to sea-level rise. Sea level rises because water expands as it is warmed and because water from melting glaciers and ice sheets is added to the oceans. Since 1993 satellite observations have permitted more precise calculations of global sea level rise, now estimated to be $3.1 \pm$ 0.7 millimeters per year over the period 1993 to 2003 . Some previous decades displayed similarly fast rates, and longer satellite records will be needed to determine unambiguously whether sea-level rise is 
accelerating. Substantial reductions in the extent of Arctic sea ice since 1978 (2.7 \pm 0.6 percent per decade in the annual average, $7.4 \pm 2.4$ percent per decade for summer), increases in permafrost temperatures and reductions in glacial extent globally and in Greenland and Antarctic ice sheets have also been observed in recent decades. Unfortunately, many of these quantities were not well monitored until recent decades, so the starting points of their records vary.

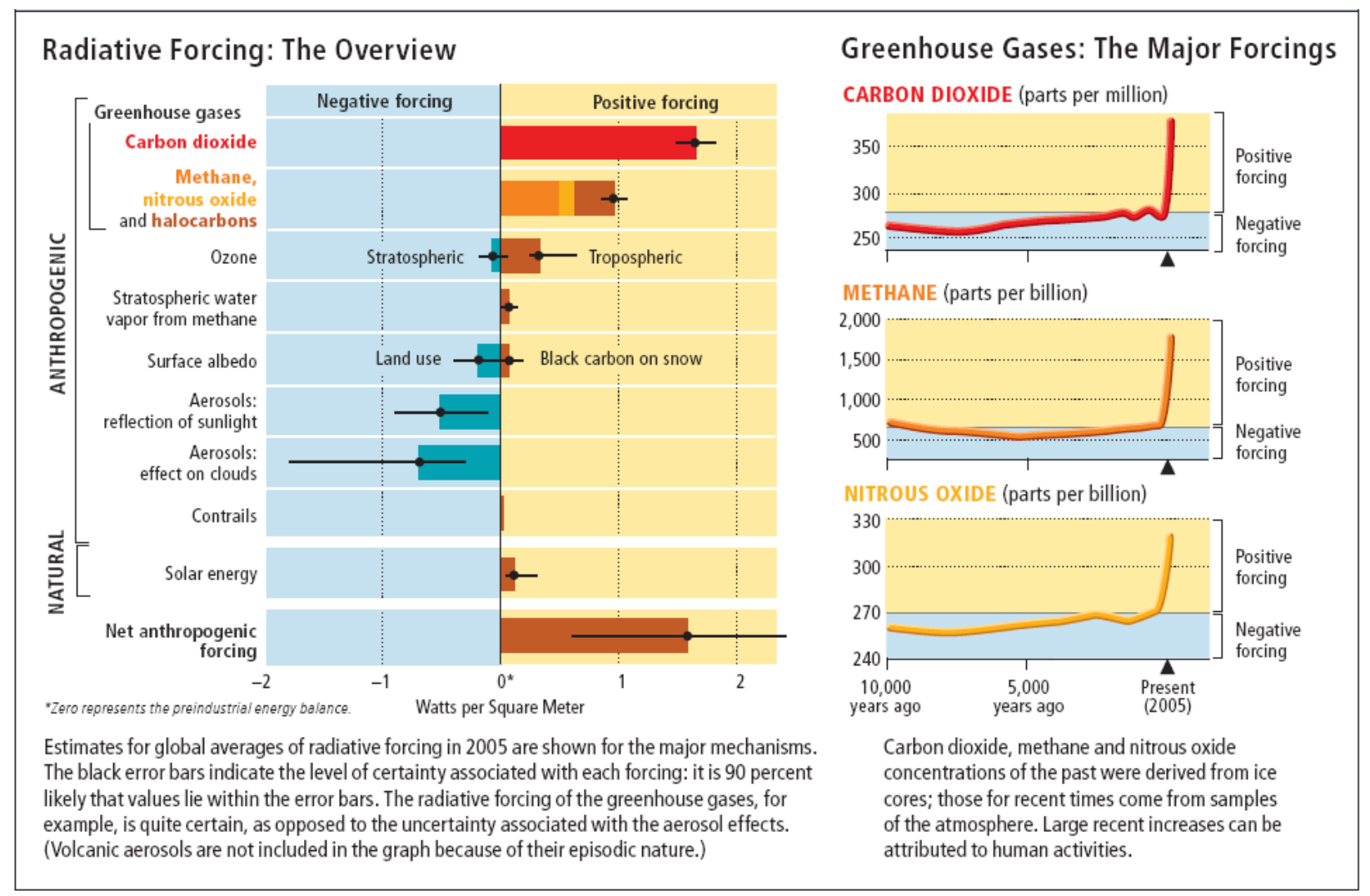

Hydrological changes are broadly consistent with warming as well. Water vapor is the strongest greenhouse gas; unlike other greenhouse gases, it is controlled principally by temperature. It has generally increased since at least the 1980s. Precipitation is very variable locally but has increased in several large regions of the world, including eastern North and South America, northern Europe, and northern and central Asia. Drying has been observed in the Sahel, the Mediterranean, southern Africa and parts of southern Asia. Ocean salinity can act as a massive rain gauge. Near-surface waters of the oceans have generally freshened in middle and high latitudes, while they have become saltier in lower latitudes, consistent with changes in large-scale patterns of precipitation. 


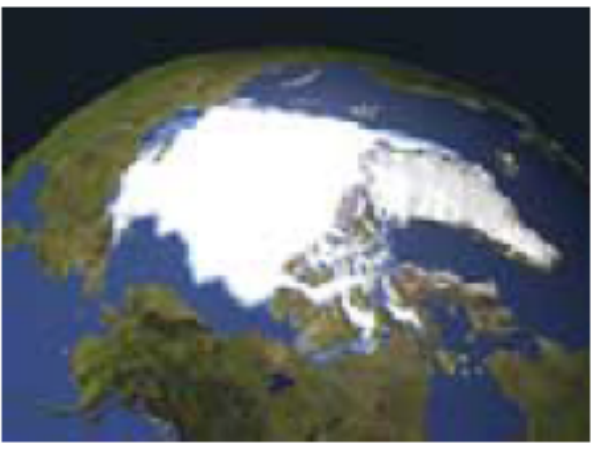

Arctic sea ice, 1979



Arctic sea ice, 2005

Reconstructions of past climate-paleoclimate - from tree rings and other proxies provide important additional insights into the workings of the climate system with and without human influence. They indicate that the warmth of the past half a century is unusual in at least the previous 1,300 years. The warmest period between A.D. 700 and 1950 was probably A.D. 950 to 1100, which was several tenths of a degree $\mathrm{C}$ cooler than the average temperature since 1980.

\section{Attribution of Observed Changes}

Although confidence is high both that human activities have caused a positive radiative forcing and that the climate has actually changed, can we confidently link the two? This is the question of attribution: Are human activities primarily responsible for observed climate changes, or is it possible they result from some other cause, such as some natural forcing or simply spontaneous variability within the climate system? The 2001 IPCC report concluded it was likely (more than 66 percent probable) that most of the warming since the mid-20th century was attributable to humans. The 2007 report goes significantly further, upping this to very likely (more than 90 percent probable).

The source of the extra confidence comes from a multitude of separate advances. For a start, observational records are now roughly five years longer, and the global temperature increase over this period has been largely consistent with IPCC projections of greenhouse gas-driven warming made in previous reports dating back to 1990. In addition, changes in more aspects of the climate have been considered, such as those in atmospheric circulation or in temperatures within the ocean. Such changes paint a consistent and now broadened picture of human intervention. Climate models, which are central to attribution studies, have also improved and are able to represent the current climate and that of the recent past with considerable fidelity. Finally, some important apparent inconsistencies noted in the observational record have been largely resolved since the last report.

The most important of these was an apparent mismatch between the instrumental surface temperature record (which showed significant warming over recent decades, consistent with a human impact) and the balloon and satellite atmospheric records (which showed little of the expected warming). Several new studies of the satellite and balloon data have now largely resolved this discrepancy-with consistent warming found at the surface and in the atmosphere. 


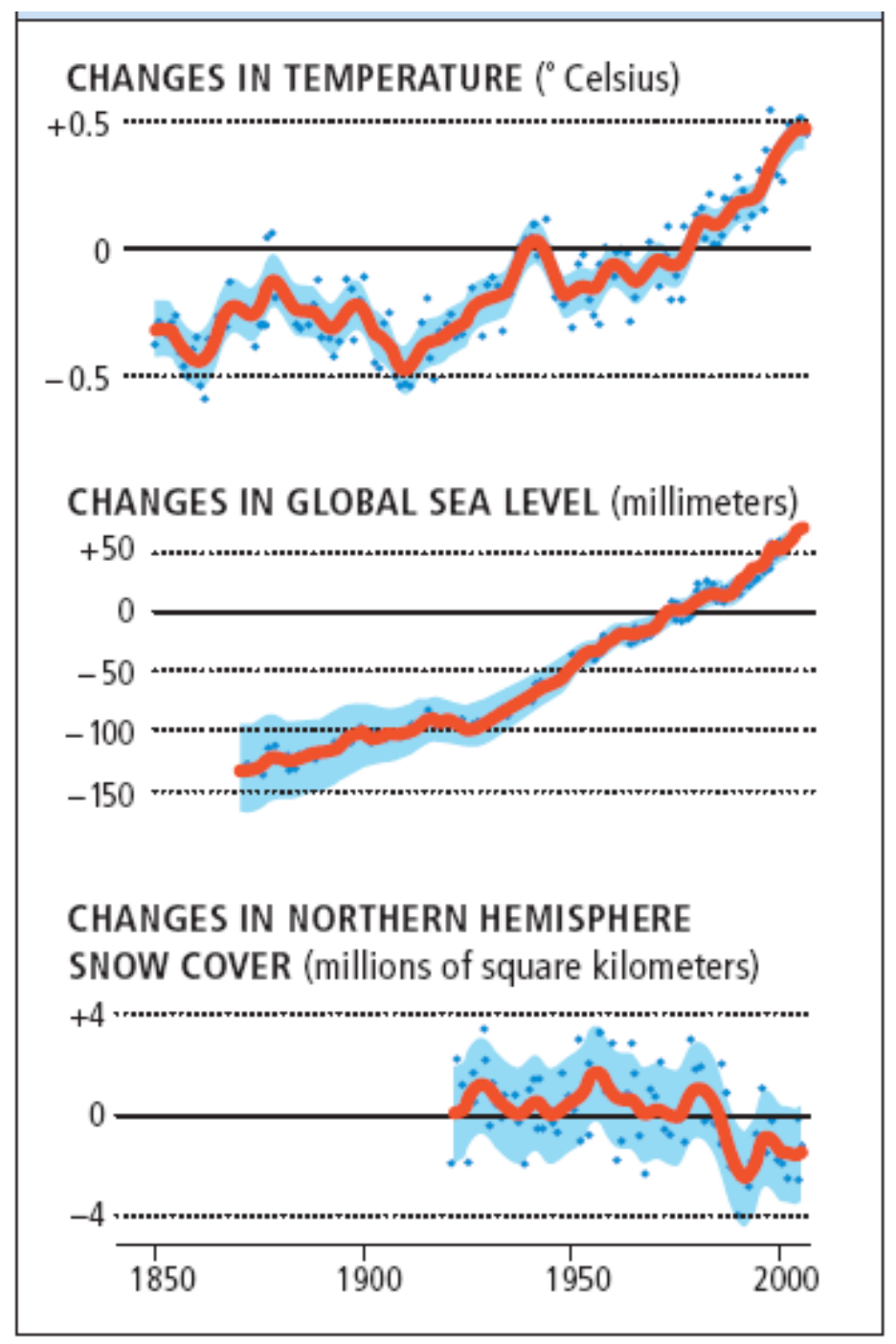

An experiment with the real world that duplicated the climate of the 20th century with constant (rather than increasing) greenhouse gases would be the ideal way to test for the cause of climate change, but such an experiment is of course impossible. So scientists do the next best thing: they simulate the past with climate models.

Two important advances since the last IPCC assessment have increased confidence in the use of models for both attribution and projection of climate changes. The first is the development of a comprehensive, closely coordinated ensemble of simulations from 18 modeling groups around the world for the historical and future evolution of the earth's climate. Using many models helps to quantify the effects of uncertainties in various climate processes on the range of model simulations. Although some processes are well understood and well represented by physical equations (the flow of the atmosphere and ocean or the propagation of sunlight and heat, for example), some of the most critical components of the climate system are less well understood, such as clouds, ocean eddies and transpiration by vegetation. Modelers approximate these components using simplified representations called parameterizations. The principal reason to develop a multimodel ensemble for the IPCC assessments is to understand how this lack of 
certainty affects attribution and prediction of climate change. The ensemble for the latest assessment is unprecedented in the number of models and experiments performed.

The second advance is the incorporation of more realistic representations of climate processes in the models. These processes include the behavior of atmospheric aerosols, the dynamics (movement) of sea ice, and the exchange of water and energy between the land and the atmosphere. More models now include the major types of aerosols and the interactions between aerosols and clouds.

When scientists use climate models for attribution studies, they first run simulations with estimates of only "natural" climate influences over the past 100 years, such as changes in solar output and major volcanic eruptions. They then run models that include human-induced increases in greenhouse gases and aerosols. The results of such experiments are striking [see box below]. Models using only natural forcings are unable to explain the observed global warming since the mid-20th century, whereas they can do so when they include anthropogenic factors in addition to natural ones. Large-scale patterns of temperature change are also most consistent between models and observations when all forcings are included.

Two patterns provide a fingerprint of human influence. The first is greater warming over land than ocean and greater warming at the surface of the sea than in the deeper layers. This pattern is consistent with greenhouse gas- induced warming by the overlying atmosphere: the ocean warms more slowly because of its large thermal inertia. The warming also indicates that a large amount of heat is being taken up by the ocean, demonstrating that the planet's energy budget has been pushed out of balance. A second pattern of change is that while the troposphere (the lower region of the atmosphere) has warmed, the stratosphere, just above it, has cooled. If solar changes provided the dominant forcing, warming would be expected in both atmospheric layers. The observed contrast, however, is just that expected from the combination of green house gas increases and stratospheric ozone decreases. This collective evidence, when subjected to careful statistical analyses, provides much of the basis for the increased confidence that human influences are behind the observed global warming. Suggestions that cosmic rays could affect clouds, and thereby climate, have been based on correlations using limited records; they have generally not stood up when tested with additional data, and their physical mechanisms remain speculative. 


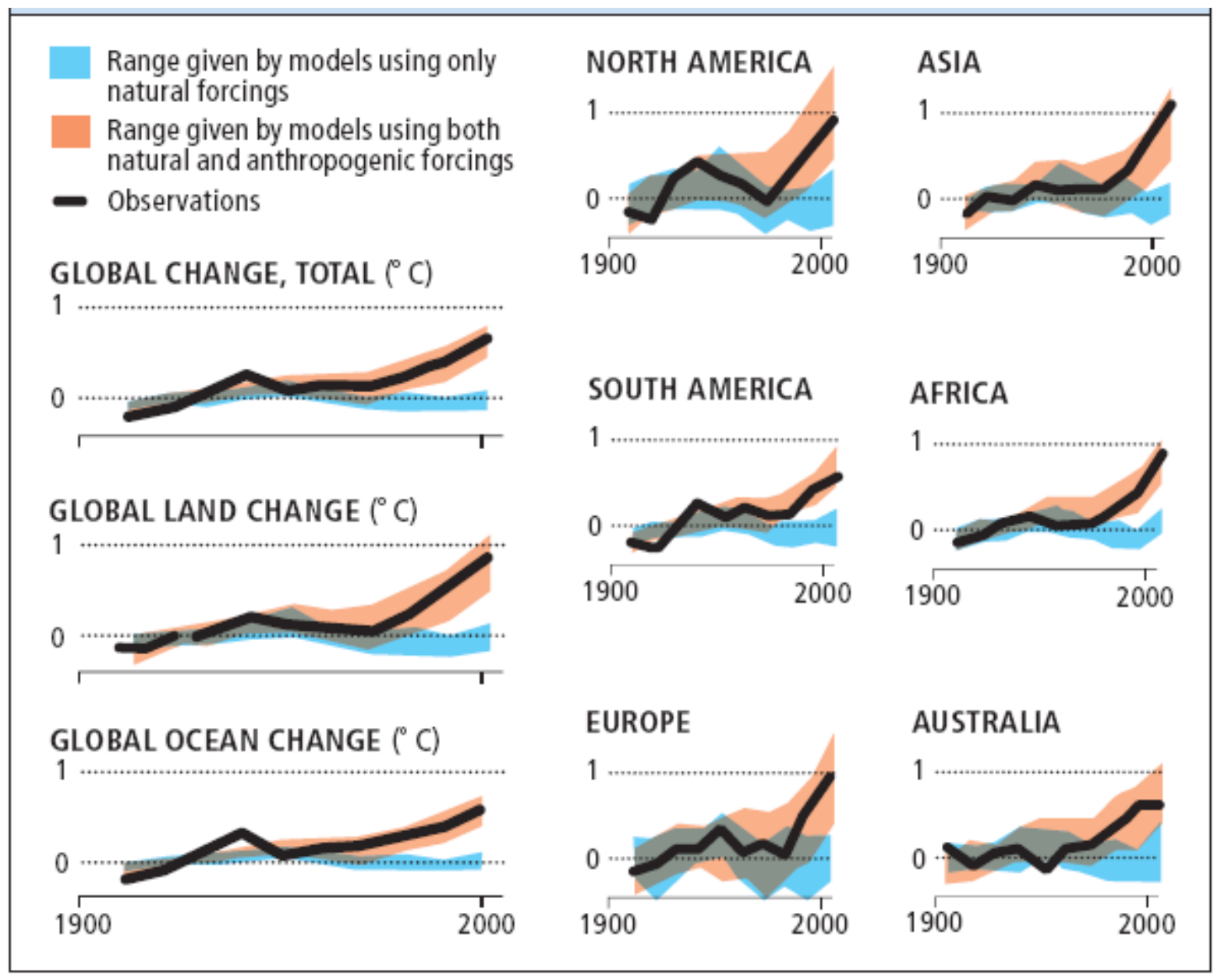

What about at smaller scales? As spatial and temporal scales decrease, attribution of climate change becomes more difficult. This problem arises because natural small-scale temperature variations are less "averaged out" and thus more readily mask the change signal. Never the less, continued warming means the signal is emerging on smaller scales. The report has found that human activity is likely to have influenced temperature significantly down to the continental scale for all continents except Antarctica.

Human influence is discernible also in some extreme events such as unusually hot and cold nights and the incidence of heat waves. This does not mean, of course, that individual extreme events (such as the 2003 European heat wave) can be said to be simply "caused" by human induced climate change—usually such events are complex, with many causes. But it does mean that human activities have, more likely than not, affected the chances of such events occurring.

\section{Projections of Future Changes}

How will climate change over the 21 st century? This critical question is addressed using simulations from climate models based on projections of future emissions of greenhouse gases and aerosols. The simulations suggest that, for greenhouse gas emissions at or above current rates, changes in climate will very likely be larger than the changes already observed during the 20th century. Even if emissions were immediately reduced enough to stabilize greenhouse gas concentrations at current levels, climate change 
would continue for centuries. This inertia in the climate results from a combination of factors. They include the heat capacity of the world's oceans and the millennial timescales needed for the circulation to mix heat and carbon dioxide throughout the deep ocean and thereby come into equilibrium with the new conditions.

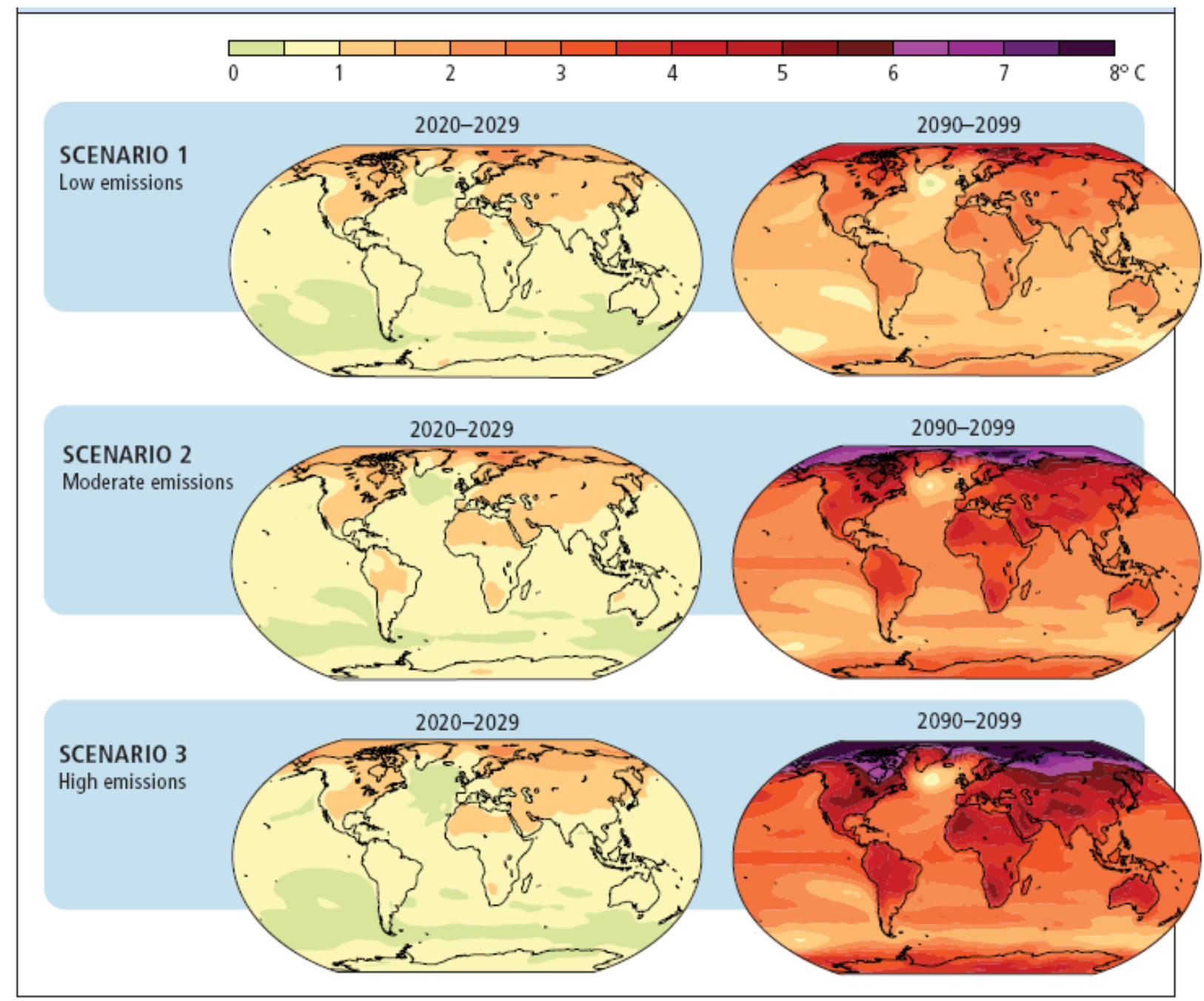

To be more specific, the models project that over the next 20 years, for a range of plausible emissions, the global temperature will increase at an average rate of about 0.2 degree $\mathrm{C}$ per decade, close to the observed rate over the past 30 years. About half of this near-term warming represents a "commitment" to future climate change arising from the inertia of the climate system response to current atmospheric concentrations of greenhouse gases.

The long-term warming over the 21st century, however, is strongly influenced by the future rate of emissions, and the projections cover a wide variety of scenarios, ranging from very rapid to more modest economic growth and from more to less dependence on fossil fuels. The best estimates of the increase in global temperatures range from 1.8 to 4.0 degrees $\mathrm{C}$ for the various emission scenarios, with higher 
emissions leading to higher temperatures. As for regional impacts, projections indicate with more confidence than ever before that these will mirror the patterns of change observed over the past 50 years (greater warming over land than ocean, for example) but that the size of the changes will be larger than they have been so far.

The simulations also suggest that the removal of excess carbon dioxide from the atmosphere by natural processes on land and in the ocean will become less effi cient as the planet warms. This change leads to a higher percentage of emitted carbon dioxide remaining in the atmosphere, which then further accelerates global warming. This is an important positive feedback on the carbon cycle (the exchange of carbon compounds throughout the climate system). Although models agree that carbon-cycle changes represent a positive feedback, the range of their responses remains very large, depending, among other things, on poorly understood changes in vegetation or soil uptake of carbon as the climate warms. Such processes are an important topic of ongoing research.

The models also predict that climate change will affect the physical and chemical characteristics of the ocean. The estimates of the rise in sea level during the 21 st century range from about 30 to 40 centimeters, again depending on emissions. More than 60 percent of this rise is caused by the thermal expansion of the ocean. Yet these model-based estimates do not include the possible acceleration of recently observed increases in ice loss from the Greenland and Antarctic ice sheets. Although scientific understanding of such effects is very limited, they could add an additional 10 to 20 centimeters to sealevel rises, and the possibility of significantly larger rises cannot be excluded. The chemistry of the ocean is also affected, as the increased concentrations of atmospheric carbon dioxide will cause the ocean to become more acidic.

Some of the largest changes are predicted for polar regions. These include significant increases in highlatitude land temperatures and in the depth of thawing in permafrost regions and sharp reductions in the extent of summer sea ice in the Arctic basin. Lower latitudes will likely experience more heat waves, heavier precipitation, and stronger (but perhaps less frequent) hurricanes and typhoons. The extent to which hurricanes and typhoons may strengthen is uncertain and is a subject of much new research.

Some important uncertainties remain, of course. For example, the precise way in which clouds will respond as temperatures increase is a critical factor governing the overall size of the projected warming. The complexity of clouds, however, means that their response has been frustratingly difficult to pin down, and, again, much research remains to be done in this area.

We are now living in an era in which both humans and nature affect the future evolution of the earth and its inhabitants. Unfortunately, the crystal ball provided by our climate models becomes cloudier for predictions out beyond a century or so. Our limited knowledge of the response of both natural systems and human society to the growing impacts of climate change compounds our uncertainty. One result of global warming is certain, however. Plants, animals and humans will be living with the consequences of climate change for at least the next thousand years.

\section{Acknowledgment}

The first author wishes to acknowledge the support provided by the Director, Office of Science, Office of Biological and Environmental Research, Climate Change Research Division, of the U.S. Department of Energy under Contract No. DE-AC02-05CH11231. 